Ricardo D'Oliveira Vieira

\title{
Estudo comparativo entre os custos dos tratamentos clínico, cirúrgico ou percutâneo em portadores de doença multiarterial coronária estável - 5 anos de seguimento
}

Tese apresentada à Faculdade de Medicina da Universidade de São Paulo para obtenção do título de Doutor em Ciências

Programa de: Cardiologia

Orientador: Prof. Dr. Whady Armindo Hueb

São Paulo 
Dados Internacionais de Catalogação na Publicação (CIP)

Preparada pela Biblioteca da

Faculdade de Medicina da Universidade de São Paulo

Creprodução autorizada pelo autor

Vieira, Ricardo D’Oliveira

Estudo comparativo entre os custos dos tratamentos clínico, cirúrgico ou percutâneo em portadores de doença multiarterial coronária estável - 5 anos de seguimento / Ricardo D’Oliveira Vieira. -- São Paulo, 2013.

Tese(doutorado)--Faculdade de Medicina da Universidade de São Paulo. Programa de Cardiologia.

Orientador: Whady Armindo Hueb.

Descritores: 1.Estudo clínico controlado randomizado 2.Doença da artéria coronariana 3.Revascularização miocárdica 4.Angioplastia coronária transluminal percutânea 5.Stents 6.Avaliação de custo-efetividade

USP/FM/DBD-054/13 


\section{Dedicatória}


À minha esposa, Joana Luisa Cylleno Vieira, pessoa indescritível, exemplo de amor e dedicação incondicional, cúmplice em todos meus projetos. Meus agradecimentos pela compreensão nos momentos de minha ausência, e por ter realizado nosso sonho maravilhoso que são nossos filhos.

Aos meus filhos, Francisco, Pedro e Gabriel Cylleno Vieira, fontes de inspiração da minha vida, crianças maravilhosas que me dão alegria, incentivo e equilíbrio, meus "anjos de guarda".

Ao meu pai, Roberto Nascimento Vieira, no qual me espelho por sua capacidade profissional e pelo exemplo de caráter e formação moral. Minha admiração por sua dedicação à nossa família e pela oportunidade de exercermos juntos a nossa profissão, sempre estimulando o meu crescimento profissional.

À minha mãe, Carmen Lúcia D’Oliveira Vieira, pela enorme importância na minha vida, participando ativamente dos meus estudos, e por toda atenção, amizade, carinho e conforto nos momentos angustiantes.

Aos meus irmãos, Letícia, Renato e Roberto D'Oliveira Vieira, pelo amor, carinho, cumplicidade e presença em toda minha trajetória de vida. Exemplos de determinação e perseverança. 
Ao cunhado, Jorge Andion Torreão, um grande amigo, pelo exemplo de superação, companheirismo, e pela grande ajuda na minha prática médica atual.

Aos meus cunhados, Katiusha de Cerqueira Abreu, Natália Oliveira e Silva, Cyel Cylleno Neto e Ana Letícia Cylleno, pelo prazer e orgulho de tê-los como parte de minha família.

Aos meus sogros, Cyel Cylleno Filho e Edna Maria da Silva Cylleno, pessoas maravilhosas que contribuíram diretamente com a minha realização pessoal.

Ao meu tio Felipe Nascimento Vieira, exemplo de padrinho, uma pessoa pacificadora, sempre presente e solícita, de quem gosto e admiro muito.

A minha avó e madrinha Raimunda Nascimento Vieira, a quem tenho muito amor e carinho.

A minha avó Maria Talon D’Oliveira, por todo amor, cuidado e prestatividade. Sinto saudade.

À Nilda Cardoso dos Santos, pela ajuda para criar um ambiente tranquilo, facilitando escrever esta tese. 
Agradecimentos 
Ao meu orientador, Professor Doutor Whady Armindo Hueb. O meu agradecimento pelo privilégio de participar do MASS, centro de excelência em pesquisa clínica, e por poder realizar esta tese. Uma pessoa que admiro como modelo de profissionalismo, honestidade e de ensinamento. A minha eterna admiração pelo seu trabalho, e gratidão pelo convívio profissional.

Ao Dr. Desidério Favarato, pelos ensinamentos e auxílio para a análise estatística deste projeto, sendo de extrema importância para a conclusão deste trabalho.

Ao amigo e colega do MASS, Dr. Eduardo Gomes Lima, pelas inúmeras ajudas prestadas e disponibilidade em todos os momentos difíceis, desde o período da residência médica.

Aos meus amigos e colegas do MASS, Dra. Cibele Larrosa Garzillo, Dra. Rosa Maria Rahmi Garcia e Dr. Paulo Cury Rezende, pela amizade, oportunidade de convívio, apoio na execução deste projeto e pelos conselhos durante a pós-graduação.

Ao Dr. Alexandre Costa Pereira, pelo auxílio nas diversas análises estatísticas realizadas durante a pós-graduação.

Às enfermeiras e amigas do MASS, Teryo Nakano, Priscila Borges Miyamoto, Myrthes Emy Takiuti e Ana Luiza de Oliveira Carvalho, pelo auxílio na assistência humanizada dos pacientes, e pelo convívio prazeroso neste período.

À secretária e amiga do MASS, Laura Caringe, pelos diversos auxílios prestados e pela paciência extraordinária. Não tem como retribuir a sua dedicação. 
Às secretárias e amigas do MASS, Eliana Olimpio Lima e Marcela Francisca da Silva, pelo apoio na execução deste projeto, pelos auxílios prestados e convívio harmonioso.

À coordenação de pós-graduação do InCor, Neusa Rodrigues Dini e Juliana Lattari Sobrinho Pagni, pela atenção, acolhimento e profissionalismo.

Aos queridos amigos, Odilson Marcos Silvestre, Luciano Dias Nascimento e Márcia de Andrade Reis Nascimento, pelo apoio que deram neste momento da minha vida. Poder contar com a ajuda de vocês foi uma grande sorte, alegria e motivo de tranqüilidade.

À professora de inglês e amiga, Helen Susan Stavros Castelhano, pela constante ajuda nos desafios internacionais e pela correção ortográfica desta tese.

Aos pacientes, foco da minha profissão, que tornaram este estudo possível, e para os quais, espero que este trabalho contribua no futuro. 


\section{NORMATIZAÇÃO ADOTADA}

Esta tese está de acordo com as seguintes normas, em vigor no momento desta publicação:

Referências: adotado de International Committee of Medical Journals Editors (Vancouver)

Universidade de São Paulo. Faculdade de Medicina. Serviço de Biblioteca e Documentação. Guia de apresentação de dissertações, teses e monografias. Elaborado por Anneliese Carneiro da Cunha, Maria Júlia de A. L. Freddi, Maria F. Crestana, Marinalva de Souza Aragão, Suely Campos Cardoso, Valéria Vilhena. $3^{\mathrm{a}}$ ed. São Paulo: Serviço de Biblioteca e Documentação; 2011.

Abreviaturas dos títulos dos periódicos, de acordo com o List of Journals Indexed in Index Medicus. 
Sumário 
Lista de abreviaturas e siglas...................................................... xiii

Lista de figuras............................................................................... xiv

Lista de tabelas............................................................................. XV

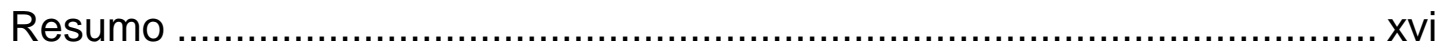

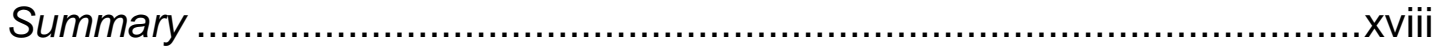

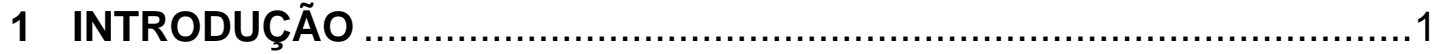

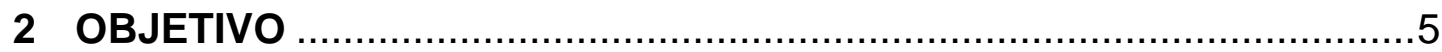

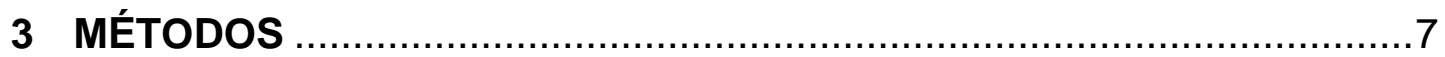

3.1 Critérios para inclusão........................................................ 8

3.2 Critérios para não inclusão .................................................. 9

3.3 Intervenções terapêuticas ....................................................10

3.4 Seguimento clínico .......................................................11

3.5 Custos hospitalares .........................................................

3.5.1 Tratamento medicamentoso .......................................13

3.5.2 Tratamento cirúrgico .................................................13

3.5.3 Tratamento percutâneo..............................................14

3.5.4 Admissão de pacientes .............................................15

3.5.5 Transporte dos pacientes ..............................................15

3.5.6 Sala de operação .........................................................16

3.5.7 Sala de terapia intensiva ............................................... 16

3.6 Custo do seguimento clínico durante cinco anos .........................17

3.7 Análise econômica ............................................................. 18

3.8 Custo-efetividade .............................................................. 19

3.9 Análise estatística ...........................................................21

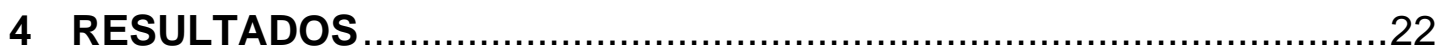

4.1 Tratamento cirúrgico........................................................26

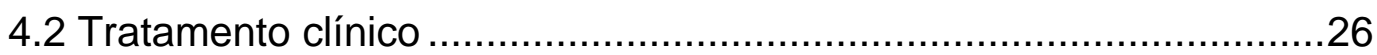

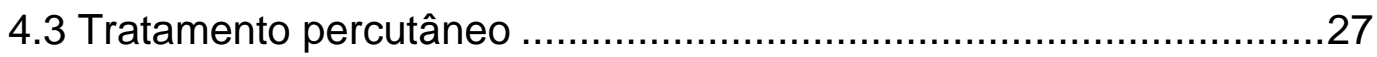

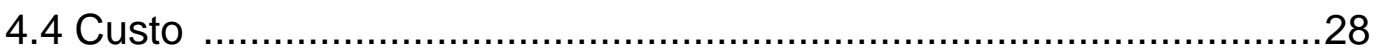

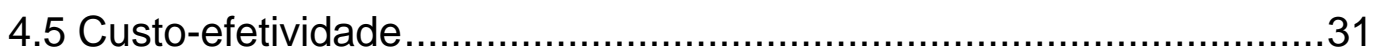

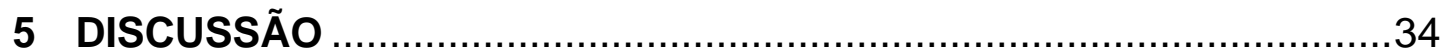

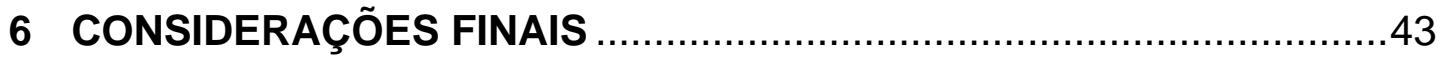

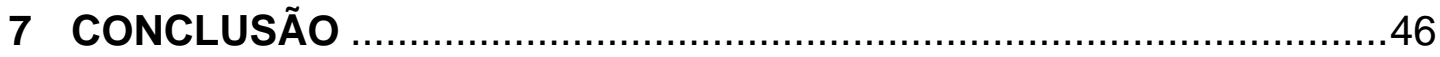

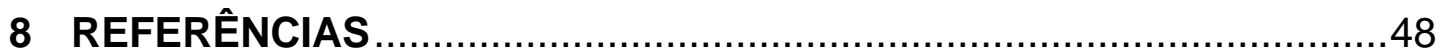


Listas

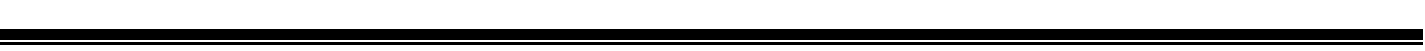




\section{ABREVIATURAS E SIGLAS}

\begin{tabular}{|c|c|}
\hline $\mathrm{Al}-$ & angina instável \\
\hline ANOVA - & ANalysis Of VAriance \\
\hline ARTS - & Arterial Revascularization Therapies Study \\
\hline BARI - & Bypass Angioplasty Revascularization Investigation \\
\hline BARI 2D - & $\begin{array}{l}\text { Bypass Angioplasty Revascularization Investigation } \\
\text { Diabetes }\end{array}$ \\
\hline CABG - & coronary artery bypass graft surgery \\
\hline CAD - & coronary artery disease \\
\hline CCS - & Canadian Cardiovascular Society \\
\hline CE - & custo-efetividade \\
\hline COURAGE - & $\begin{array}{l}\text { Clinical Outcomes Utilizing Revascularization and Aggressive } \\
\text { Drug }\end{array}$ \\
\hline CRM - & cirurgia de revascularização miocárdica \\
\hline CK-MB - & creatinofosfoquinase-MB \\
\hline DAC - & doença arterial coronariana \\
\hline ECG - & eletrocardiograma \\
\hline ECR - & ensaio clínico randomizado \\
\hline FMUSP - & Faculdade de Medicina da Universidade de São Paulo \\
\hline HC/FMUSP - & $\begin{array}{l}\text { Hospital das Clínicas da Faculdade de Medicina da } \\
\text { Universidade de São Paulo }\end{array}$ \\
\hline IAM - & infarto agudo do miocárdio \\
\hline ICP - & intervenção coronária percutânea \\
\hline MASS II - & Second Medicine, Angioplasty, or Surgery Study \\
\hline $\mathrm{PCl}-$ & percutaneous coronary intervention \\
\hline QALY - & quality- adjusted life-year \\
\hline RITA-2 - & Second Randomized Intervention Treatment of Angina \\
\hline SDRA - & síndrome do desconforto respiratório do adulto \\
\hline TC - & tratamento clínico \\
\hline 2 & teste de qui-quadrado \\
\hline
\end{tabular}


FIGURAS

Figura 1 - Custo cumulativo das 3 estratégias terapêuticas ....................28

Figura 2 - Composição dos custos nas 3 estratégias terapêuticas............29

Figura 3 - Proporção do custo cumulativo das 3 estratégias terapêuticas.......................................................... 30

Figura 4 - Custo cumulativo livre de evento em 5 anos de seguimento ....32

Figura 5 - Custo cumulativo livre de evento e angina em 5 anos de seguimento. 
TABELAS

Tabela 1 - Características demográficas, laboratoriais e clinicas dos pacientes.

Tabela 2 - Eventos cardiovasculares adversos - 5 anos de seguimento. .25

Tabela 3 - Sobrevida livre de evento e proporção livre de angina. 31 
Resumo

(20) 
Vieira RD. Estudo comparativo entre os custos dos tratamentos clínico, cirúrgico ou percutâneo em portadores de doença multiarterial coronária estável - 5 anos de seguimento [tese]. São Paulo: Faculdade de Medicina, Universidade de São Paulo, 2013.

INTRODUÇÃO: As principais opções terapêuticas para a doença multiarterial coronária incluem cirurgia de revascularização miocárdica (CRM), intervenção coronária percutânea (ICP) e tratamento clínico (TC). Essas três estratégias terapêuticas apresentam eficácia similar em determinados subgrupos de pacientes. No presente momento, estudos direcionados à análise econômica são escassos, e contemplam, principalmente, os custos comparativos entre as intervenções cirúrgica e percutânea. OBJETIVOS: Analisar, prospectivamente, o custo comparativo das três formas terapêuticas da doença multiarterial coronária estável, durante cinco anos de seguimento. MÉTODOS: Foi computado o custo terapêutico global de 611 pacientes do ensaio clínico The Second Medicine, Angioplasty, or Surgery Study (MASS II), baseado na remuneração provida pelo sistema de saúde suplementar do Instituto do Coração do HC/FMUSP, tomando-se os valores em moeda nacional corrente. Realizou-se, posteriormente, análise de custo-efetividade para o tempo livre de eventos clínicos e o tempo livre de eventos acrescido de tempo livre de angina. RESULTADOS: O TC apresentou 3.79 e 2.07 QALY (quality-adjusted lifeyears); o ICP apresentou 3.59 e 2.77 QALY; e o CRM apresentou 4.4 e 2.81 QALY, respectivamente, para sobrevida livre de eventos e sobrevida livre de eventos e angina. Os custos para sobrevida livre de eventos foram $R \$$ $16.327,80$ para TC, $\mathrm{R} \$ 35.940,60$ para ICP e $\mathrm{R} \$ 32.873,40$ para CRM. A análise pareada dos custos para sobrevida livre de eventos mostrou que houve diferença significante favorecendo TC contra ICP $(P<0,01)$, e em comparação com CRM $(P<0,01)$; e CRM versus ICP $(P=0,01)$. Os custos para sobrevida livre de eventos e angina foram $R \$ 29.795,40, R \$ 46.495,80$ e $\mathrm{R} \$ 44.305,20$, respectivamente. A comparação pareada dos custos livres de eventos mais livres de angina demonstrou que houve diferença significante favorecendo TC contra ICP $(P=0,04)$, e em comparação com CRM $(P<0,001)$. Não houve diferença entre CRM e ICP $(P>0,05)$. CONCLUSÃO: A análise comparativa entre as diferentes opções terapêuticas desta amostra revelou que TC foi mais custo-efetivo que CRM, e esta, por sua vez, mais custo-efetivo que ICP.

Descritores: 1.Estudo clínico controlado randomizado 2.Doença da artéria coronariana 3.Revascularização miocárdica 4.Angioplastia coronária transluminal percutânea 5.Stents 6.Avaliação de custo-efetividade. 
Summary 
Vieira RD. Comparative cost analysis for surgical, angioplasty, or medical therapeutics for coronary artery disease - 5-year follow-up [thesis]. São Paulo: "Faculdade de Medicina, Universidade de São Paulo", 2013.

BACKGROUND: The therapeutic options for multivessel coronary artery disease are coronary artery bypass graft surgery (CABG), percutaneous coronary intervention $(\mathrm{PCl})$, or medical treatment alone (MT). These three therapeutic strategies present similar efficacy for specific subgroups. At the present moment, economic outcome trials are scant, and contemplate comparative cost between surgical or percutaneous intervention. OBJECTIVE: To analyze, prospectively, the comparative cost from three therapeutic strategies in multivessel coronary artery disease, at 5-year of follow-up. METHODS: We analyzed cumulative costs of 611 patients from clinical trial The Second Medicine, Angioplasty, or Surgery Study (MASS II). The economic analysis is based on remuneration provided by the supplementary health system of the Heart Institute of the Clinical Hospital of FMUSP, expressing these values in Brazilian currency. It was compared to the cumulative costs of each therapeutic strategy in the 5-year follow-up period. A cost-effectiveness analysis was then conducted for event-free survival and event plus angina-free survival. Cost-effectiveness analysis was performed by quality-adjusted life- year (QALY) analysis. RESULTS: Respectively, for event-free survival and event plus angina-free survival, MT presented 3.79 quality-adjusted life-years (QALY) and 2.07 QALY; PCI presented 3.59 and 2.77 QALY; and CABG demonstrated 4.4 and 2.81 QALY. The event-free costs were R\$ 16327.80 for MT; R\$ 35940.60 for $\mathrm{PCl}$; and $\mathrm{R} \$ 32873.40$ for CABG. The paired comparison of the event-free costs showed that there was a significant difference favoring MT versus $\mathrm{PCl}$ $(P<0.01)$ and versus CABG $(P<0.01)$ and CABG versus PCl $(P=0.01)$. The event-free plus angina-free costs were $R \$ 29795.40, R \$ 46495.80$ e $\mathrm{R} \$ 44305.20$, respectively. The paired comparison of the event-free plus angina-free costs showed that there was a significant difference favoring MT versus $\mathrm{PCl}(P=0.04)$, and versus CABG $(P<0.001)$; there was no difference between CABG and $\mathrm{PCI}(P>0.05)$. CONCLUSION: The comparative analysis among the different therapeutic strategies demonstrated that MT was more cost-effective than CABG, and this than PCl.

Descriptors: 1.Randomized clinical trial 2.Coronary artery disease 3.Myocardial revascularization 4.Percutaneous transluminal coronary angioplasty 5.Stents 6.Cost-effectiveness analysis. 


\section{Introdução}


A cirurgia de revascularização miocárdica (CRM) é reconhecida nos países ocidentais como opção terapêutica eficaz no tratamento dos sintomas da doença arterial coronária (DAC) $)^{1,2}$ bem como na prevenção de infarto e/ou morte em determinados subgrupos de pacientes ${ }^{3-5}$. Esses benefícios, entretanto, vêm acompanhados de significativos índices de morbidade intrahospitalar ${ }^{6-10}$, com subsequente aumento de utilização de recursos financeiros $^{11}$, além do aumento da permanência hospitalar ${ }^{12}$. Tratamentos alternativos com os mesmos objetivos clínicos, tais como a intervenção coronária percutânea (ICP) utilizando o cateter balão, foram desenvolvidos para reduzir a morbidade hospitalar ${ }^{13}$, com consequente diminuição de internação hospitalar ${ }^{14}$, e também os custos dos procedimentos ${ }^{15,16}$. Contudo, a par os benefícios clínicos, constatou-se que esse procedimento veio acompanhado de alto índice de reestenose das artérias sob tratamento, com consequente aumento dos custos hospitalares ${ }^{17}$. Por outro lado, os avanços tecnológicos objetivando diminuir complicações clínicas decorrentes dessa técnica permitiram o surgimento de stents de várias especificações, tais como os revestidos com drogas antiproliferativas ${ }^{18}$. Isso contribuiu para a diminuição das reestenoses, mas implicou um aumento dos custos finais do tratamento ${ }^{19,20}$.

Estudos direcionados a avaliar os custos das intervenções coronárias percutâneas usando stents convencionais revelaram que, comparada com a cirurgia de revascularização miocárdica, a intervenção cirúrgica aumentava 
significativamente os custos do tratamento ${ }^{21,22}$. Contudo, quando o uso de stents farmacológicos foi comparado com a cirurgia de revascularização miocárdica, observou-se inversão dessa vantagem econômica. O novo dispositivo encareceu sobremaneira a aplicação desse recurso no tratamento da DAC, comparado à cirurgia ${ }^{23,24}$.

Por outro lado, opções conservadoras para o tratamento dessa enfermidade, em determinados subgrupos de pacientes, permitem manter a condição por um longo período de seguimento, sem a necessidade de intervenções mecânicas, o que implica diminuição dos custos financeiros, governamentais ou privados.

Essa aparente vantagem econômica pode encobrir os recursos aplicados no seguimento de longo prazo, seja com exames repetitivos, intervenções clínicas menores, eventos clínicos que impliquem internações hospitalares, ou até intervenções percutâneas ou cirúrgicas emergenciais. Com isso, os custos do tratamento conservador poderão ser maiores que os dos tratamentos intervencionistas.

Os estudos que tratam dessas questões são escassos e contemplam, principalmente, os custos comparativos entre intervenções cirúrgicas e intervenções por cateter, ou entre diferentes próteses endovasculares ${ }^{19,25}$.

É o caso de estudos prospectivos e randomizados desenvolvidos em nosso meio em que se comparam os custos hospitalares de cirurgia de revascularização miocárdica com e sem o uso de circulação extracorpórea, indicando vantagens em determinadas condições para uma ou para outra 
técnica ${ }^{26,27}$. Além disso, dados iniciais sobre custos comparativos em seguimento de curto prazo de tratamento cirúrgico, percutâneo ou clínico, revelaram vantagem inicial para o tratamento clínico ${ }^{28}$. 
2 Objetivo 
Avaliar, prospectivamente, o custo comparativo das três formas terapêuticas para doença coronária estável, durante cinco anos de seguimento. 
3 Métodos

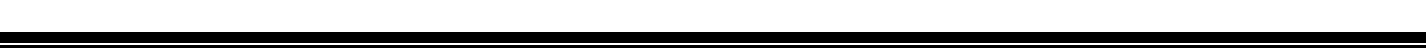


Detalhes da hipótese, modelo e métodos do protocolo de estudo The Second Medicine, Angioplasty and Surgery Study II (MASS II) foram publicados previamente ${ }^{29}$. O MASS II é um ensaio clínico randomizado, prospectivo, multidisciplinar e unicêntrico que compara a eficácia relativa da revascularização cirúrgica, percutânea ou tratamento clínico isolado, em pacientes com angina estável, doença coronária multiarterial e função ventricular esquerda preservada. O desfecho primário do estudo foi a combinação de mortalidade geral, infarto do miocárdio e a necessidade de realizar a revascularização miocárdica.

\subsection{CRITÉRIOS PARA INCLUSÃO}

Foram considerados para inclusão neste estudo pacientes com DAC que apresentassem obstrução luminal maior que $70 \%$ em pelo menos duas artérias coronárias principais, diagnosticadas visualmente por cinecoronariografia, bem como isquemia miocárdica documentada pelo teste ergométrico ou dor torácica considerada angina e graduada pela Canadian Cardiovascular Society (CCS) ${ }^{30}$.

Os pacientes foram designados para as respectivas intervenções uma vez aceitos pelo cirurgião ou pelo hemodinamicista e desde que 
considerados seguros pelo médico clínico para o tratamento conservador e, portanto, factíveis para qualquer uma das três opções terapêuticas.

Após terem assinado o termo de consentimento para o estudo, os pacientes foram encaminhados para os tratamentos clínico, cirúrgico ou através da angioplastia.

Este estudo foi desenvolvido no Instituto do Coração do Hospital das Clínicas da Faculdade de Medicina da Universidade de São Paulo (HC/FMUSP), envolvendo Unidade Clínica de Aterosclerose, Serviço de Métodos Gráficos, Laboratório de Pesquisas Clínicas, Serviço de Hemodinâmica e Divisão de Cirurgia. Obteve aprovação da Comissão Científica do Instituto do Coração, sob o número 946/94/56, e da Comissão de Ética do Hospital das Clínicas da Faculdade de Medicina da Universidade de São Paulo, sob o número 264/94/11.

\subsection{CRITÉRIOS PARA NÃO INCLUSÃO}

Os critérios para não inclusão fundamentaram-se na presença de angina instável, aneurisma ventricular que requeresse intervenção cirúrgica, disfunção ventricular com fração de ejeção < 45\%, e intervenções percutâneas ou cirúrgicas prévias. Não foram incluídos pacientes com doenças cardíacas congênitas, doença valvar, miocardiopatia ou impossibilidade de cooperar com o protocolo de estudo ou com 
acompanhamento ambulatorial. E ainda, aqueles pacientes em que se constatou presença de estenose de $50 \%$ ou mais no tronco da artéria coronária esquerda, gravidez suspeitada ou planejada ou qualquer condição que contraindicasse os tratamentos percutâneos e cirúrgicos.

\subsection{INTERVENÇÕES TERAPÊUTICAS}

Todos os pacientes foram submetidos a um completo regime terapêutico incluindo: nitratos, aspirina, betabloqueadores, bloqueadores dos canais de cálcio e inibidores da enzima conversora da angiotensina, ou uma combinação desses fármacos, a menos que fossem contraindicados. Foram prescritos ainda inibidores da hidroximetilglutaril-coenzima A redutase, assim como insulina e hipoglicemiantes orais para melhor controle de diabetes mellitus. As prescrições aplicavam-se aos três grupos terapêuticos, e os medicamentos podiam ser obtidos, sem ônus para os pacientes, no Instituto do Coração.

Para os pacientes encaminhados para tratamento percutâneo, o procedimento foi disponibilizado duas semanas depois de assinado o termo de consentimento. Recursos utilizados para terapêutica através de cateter incluíam: balão, stents, laser e aterectomia direcional. A angioplastia foi realizada de acordo com protocolo padronizado. Inibidor da glicoproteína Ilb/IIla não foi usado nesse grupo de pacientes. 
Para os pacientes encaminhados para revascularização cirúrgica, o

procedimento foi disponibilizado, igualmente, duas semanas após a assinatura do termo de consentimento. Revascularização anatômica completa foi utilizada, se tecnicamente factível, e com uso de todos os recursos, que incluíram: veia safena, artérias mamárias, artéria radial ou gastroepiploica. A técnica cirúrgica foi utilizada de acordo com o procedimento padronizado. O procedimento também incluiu cardioplegia. Não foi utilizada técnica cirúrgica sem circulação extracorpórea.

\subsection{SEGUIMENTO CLÍNICO}

Fatores adversos, bem como eventos clínicos, foram considerados a partir da data da assinatura do termo de consentimento. Os pacientes tiveram seguimento clínico ambulatorial trimestralmente, no primeiro ano de seguimento, e semestralmente, nos anos subsequentes. Todos eles, a menos que contraindicados, submeteram-se ao teste de esforço, conforme o protocolo de Bruce, na admissão do estudo e, na sequência, uma vez ao ano.

Exames laboratoriais de rotina foram realizados a cada seis meses, bem como o ECG de repouso. Estudos cintilográficos ou angiográficos estavam disponíveis para a ocorrência de sintomas anginosos de difícil controle. 
A cinecoronariografia foi realizada segundo a técnica de Sones ou Seldinger. Para a obtenção da função ventricular, foi usada a projeção oblíqua anterior direita, e o cálculo da fração de ejeção foi feito segundo a fórmula de Dodge ${ }^{31}$.

Sintomas anginosos foram graduados, segundo a gravidade, de 1 a 4, como previamente definido. Angina era considerada instável somente quando os pacientes recebiam dose plena de medicamentos anti-isquêmicos sem resposta efetiva.

Infarto do miocárdio foi considerado presente na observância de novas ondas $\mathrm{Q}$ em pelo menos duas derivações do ECG ou de sintomas de dor torácica característica, associada a aumento do nível sérico da enzima CK-MB três vezes acima do valor normal de referência. Acidente vascular encefálico foi diagnosticado mediante observação do aparecimento de déficit neurológico associado a lesão estruturalmente compatível, identificada por tomografia de crânio ou ressonância nuclear magnética. A morte foi classificada como cardíaca ou não-cardíaca.

\subsection{CUSTOS HOSPITALARES}

Os custos hospitalares foram calculados de acordo com a remuneração provida pelo Sistema de Saúde Suplementar e obtida 
diretamente da administração contábil do Instituto do Coração do HC/FMUSP, considerados os valores em moeda nacional corrente.

\subsubsection{TRATAMENTO MEDICAMENTOSO}

Por se tratar de tratamento conservador, os pacientes designados para essa modalidade terapêutica tiveram seus custos baseados principalmente nas medicações e exames periódicos de controle. Ainda que os medicamentos sejam fornecidos pelo Sistema Único de Saúde para o nosso hospital, optamos por incluí-los no cálculo dos custos terapêuticos, considerado todo o período de seguimento. Intercorrências clínicas relacionadas ao sistema cardiovascular foram consideradas eventos e admitidas como custos adicionais, assim como exames complementares realizados para elucidação das mesmas. Foram computadas ainda como custos adicionais eventuais intervenções no coração, seja por cateter ou por cirurgia, bem como exames requeridos para diagnóstico.

\subsubsection{TRATAMENTO CIRÚRGICO}

Os custos das intervenções cirúrgicas foram obtidos da mesma fonte mencionada acima para revascularização cirúrgica do miocárdio Essa cirurgia tem remuneração fixa, por paciente, independentemente do número e do tipo de enxertos. Por se tratar de procedimento de alta complexidade e 
de alto custo, a cirurgia de revascularização miocárdica tem remuneração diferenciada para cada recurso utilizado na intervenção, seja na sala de operação ou no laboratório de hemodinâmica, seja na sala de tratamento intensivo. Inclui-se o uso de órteses e próteses, tais como cateteres de Swan-Ganz, balão intra-aórtico de contrapulsação, circuito de hemodiálise, dentre outros. Dessa forma, foram calculados os gastos efetivamente aplicados em cada etapa do procedimento.

Os custos hospitalares diretos foram calculados de acordo com o Sistema de Saúde Suplementar da instituição, considerando-se os valores em moeda nacional corrente. Os custos indiretos, como taxa de administração predial, gastos com manutenção, consumo de água, luz, telefone, lavanderia, alimentação, depreciação de materiais ou da estrutura hospitalar, não foram contabilizados, por estarem presumivelmente embutidos nos custos. Já diárias extras, materiais e exames não previstos nos contratos de prestação de tratamento foram incluídos nos cálculos dos gastos.

\subsubsection{TRATAMENTO PERCUTÂNEO}

Do mesmo modo que para os custos das intervenções cirúrgicas, as informações para o tratamento percutâneo foram obtidas conforme descrição anterior. A intervenção tem remuneração fixa por paciente, na qual estão incluídos taxa de sala, equipo de fluidos intravenosos, caixa de materiais e 
roupas esterilizadas, além de cateteres e guias endovasculares bem como pessoal circulante de sala. Foram considerados à parte e contabilizados o número de artérias tratadas e o número de próteses endovasculares, tais como stents ou dispositivos descartáveis.

\subsubsection{ADMISSÃO DE PACIENTES}

Durante o período de preparo do paciente para qualquer uma das intervenções, cirurgia ou angioplastia, consideraram-se gastos com diárias hospitalares e equipes médica, de enfermagem e de fisioterapeutas. Medicamentos e exames de pré-operatório não foram calculados.

\subsubsection{TRANSPORTE DOS PACIENTES}

Por se tratar de procedimentos de rotina e possivelmente já embutidos nos cálculos hospitalares, os custos de transporte dos pacientes, como macas especiais, pessoal de transporte, incluídas enfermeiras, medicamentos, pré-anestésicos, chegada à sala de operação e preparo para a anestesia, não foram considerados. 


\subsubsection{SALA DE OPERAÇÃO}

Itens como depreciação da sala de operação, do laboratório de hemodinâmica ou de material e remuneração do pessoal circulante das salas cirúrgicas ou de hemodinâmica não foram considerados, assim como custos das equipes médica e de anestesia, por já estarem embutidos no valor final do procedimento, do mesmo modo que os custos do pessoal de instrumentação de cirurgia ou de angioplastia.

Já os hemoderivados administrados na sala de operação e na unidade de terapia intensiva ou nas enfermarias tiveram seus custos calculados. A caixa de instrumentos cirúrgicos não entrou no cálculo.

\subsubsection{SALA DE TERAPIA INTENSIVA}

Assim como para os gastos com o pré-operatório, não foram considerados os custos de transportes do paciente da sala de operação para a sala de terapia intensiva. Todavia, foram calculados os tempos de permanência do paciente na unidade, tempo de necessidade de ventilação

mecânica e uso de recursos de alta complexidade e de alto custo, como balão intra-aórtico, circuito de hemofiltração e cateter de Swan-Ganz. Esses recursos estavam disponíveis para complicações do pós-operatório. No caso de uma reoperação, o cálculo seria o de uma nova operação. 
Complicações ocorridas no pós-operatório, desde a saída da unidade de terapia intensiva até a alta hospitalar, tais como acidente cérebro vascular infarto agudo do miocárdio, infecções respiratórias e de ferida operatória, foram consideradas e calculadas de acordo com os valores definidos pelo hospital.

\subsection{CUSTO DO SEGUIMENTO CLÍNICO DURANTE CINCO ANOS}

Todos os pacientes, qualquer que fosse a modalidade terapêutica, tiveram seguimento ambulatorial agendado a cada três meses, no primeiro ano de seguimento, e a cada seis meses, nos anos subsequentes. Ao longo de todo o período, foram realizados exames laboratoriais, eletrocardiográficos de repouso e de esforço, ecocardiogramas, cintilografias e cinecoronariografias e fornecidos medicamentos para o controle de sintomas anginosos, de fatores de risco cardiovascular e de intercorrências clínicas, tais como acidente cerebrovascular, infarto agudo do miocárdio e angina instável com necessidade de intervenção. Esses itens foram computados nos cálculos. Cirurgia cardíaca adicional e intervenção coronária percutânea foram consideradas eventos e seus custos calculados como novos procedimentos para qualquer grupo terapêutico. 


\subsection{ANÁLISE ECONÔMICA}

A análise dos custos foi realizada em parceria com a Health Research and Policy Department of Stanford University School of Medicine.

A análise econômica comparou os custos cumulativos de cada estratégia terapêutica ao longo de cinco anos de seguimento. Resumidamente, os recursos analisados foram: (1) procedimento de revascularização inicial (ICP ou CRM) após a randomização; complicações intra-hospitalares dos procedimentos de revascularização; (3) consultas ambulatoriais; (4) exames complementares cardiovasculares (teste ergométrico, ecocardiograma, cintilografia miocárdica, cineangiocoronariografia); (5) hospitalização subsequente por doença cardiovascular (infarto do miocárdio, angina instável, acidente vascular encefálico e morte); (6) procedimentos de revascularização subsequentes e complicações intra-hospitalares; (7) medicamentos.

O custo de cada recurso no Instituto do Coração do HC/FMUSP é, respectivamente: $\mathrm{R} \$ 17.500,00$ por cirurgia de revascularização miocárdica (CRM); $\mathrm{R} \$ 4.500,00$ por angioplastia; $\mathrm{R} \$ 3.880,00$ por stent convencional; $\mathrm{R} \$ 10.000,00$ por hospitalização decorrente de infarto agudo do miocárdio (IAM); $\mathrm{R} \$ 9.000,00$ por hospitalização decorrente de angina instável (AI); $\mathrm{R} \$$ 6.000,00 por hospitalização decorrente de acidente vascular encefálico (AVE); $\mathrm{R} \$ 1.200,00$ por cinecoronariografia; $\mathrm{R} \$ 617,00$ por cintilografia 
miocárdica; $\mathrm{R} \$ 145,00$ por ecocardiograma; $\mathrm{R} \$ 80,00$ por teste ergométrico; $\mathrm{R} \$ 41,00$ por consulta ambulatorial.

O custo médio das complicações hospitalares dos procedimentos de revascularização é, respectivamente: $\mathrm{R} \$ 11.646,00$ por episódio de choque cardiogênico com uso de balão de contrapulsação intra-aórtico; R\$ 10.587,00 por episódio de pneumonia nosocomial ou síndrome do desconforto respiratório do adulto (SDRA); $\mathrm{R} \$ 5.293,00$ por episódio de disfunção renal aguda com necessidade de hemodiálise; $\mathrm{R} \$ 12.704,00$ por episódio de mediastinite ou osteomielite de esterno; $\mathrm{R} \$ 9.000,00$ para cada cirurgia cardíaca por sangramento e/ou tamponamento cardíaco.

Os custos de cinco anos de medicação foram baseados no estudo Arterial Revascularization Therapies Study (ARTS), sendo US\$ 5.000,00 para os pacientes submetidos a terapia de revascularização e US $\$ 6.000,00$ para os pacientes de tratamento clínico $^{32}$. Realizando a conversão monetária, com taxa $1.8 \mathrm{real} /$ dólar, o custo cumulativo da terapia medicamentosa é, respectivamente, $\mathrm{R} \$ 9.000,00$ e $\mathrm{R} \$ 10.800,00$,

\subsection{CUSTO-EFETIVIDADE}

A análise de custo-efetividade foi realizada pelo indicador QALY (quality-adjusted life-year) ${ }^{33-35}$, combinando-se evidência de efetividade e 
custos do tratamento para cada modalidade terapêutica. O QALY foi aplicado para a sobrevida livre de evento e de angina.

Para o cálculo do QALY para sobrevida livre de evento, estabelece-se uma relação entre o tempo médio para desfecho de cada estratégia terapêutica e o tempo de seguimento (cinco anos) - tempo médio para evento/5. No estudo MASS, o desfecho foi a combinação de morte, IAM ou a realização do procedimento de revascularização.

Para o cálculo do QALY para sobrevida livre de angina, divide-se a proporção livre de angina, ao término de cinco anos, em cada modalidade terapêutica, pelo período de seguimento - proporção livre de angina/5.

O custo para a sobrevida livre de evento é calculado com base na relação entre o custo cumulativo de cada terapia e o QALY livre de evento custo cumulativo/QALY livre de evento. Os valores são expressos em reais por ano de vida livre de evento. Obtém-se o custo livre de evento (R\$/ano de seguimento livre de evento) pela relação entre custos cumulativos medianos e tempo livre de evento de cada terapia, ao longo dos cinco anos de seguimento.

Para o cálculo do custo para a sobrevida livre de evento e angina, divide-se o custo bruto (cumulativo) pelo QALY livre de evento e o QALY livre de angina - custo cumulativo/QALY livre de evento $X$ QALY livre de angina. Os valores são expressos em reais por ano de vida livre de evento e angina. Obtém-se o custo livre de evento e angina ( $R$ \$ano de seguimento livre de evento e angina) pela relação entre custos cumulativos medianos e 
tempo livre de eventos e a proporção livre de angina, ao término de cinco anos de seguimento de cada terapia.

O presente estudo introduziu, assim, a análise QALY para a sobrevida livre de evento acrescido de angina.

\subsection{ANÁLISE ESTATÍSTICA}

A análise estatística foi realizada com teste $t$ e ANOVA para as variáveis de distribuição normal, ANOVA não-paramétrica para as variáveis de distribuição não-gaussiana e qui-quadrado para as variáveis categóricas. O valor de $P<0.05$ foi considerado de significância estatística. Os custos cumulativos foram comparados com base na análise de intention-to-treat. Para a análise estatística, utilizou-se o software SAS 9.1 (SAS Institute Inc, Chicago, III). 


\section{Resultados}


Entre maio de 1995 e maio de 2000, foram selecionados 2.390 pacientes que preenchiam todos os critérios clínicos e angiográficos para inclusão no estudo. Desses pacientes, 611 assinaram o termo de consentimento e foram encaminhados para a randomização, enquanto os demais 1.779 pacientes tiveram acompanhamento não-randomizado. As principais razões para a não-randomização foram a recusa de participar em um estudo randomizado ou algum impedimento para seguimento clínico sistematizado.

Dos 611 pacientes randomizados que compuseram a amostra do presente estudo para seguimento clínico a longo prazo, 205 receberam angioplastia, 203 foram encaminhados para cirurgia e 203 foram tratados clinicamente. O estado vital de todos os pacientes foi concluído em maio de 2005. O tempo mínimo de duração do estudo foi de cinco anos para todos.

Os grupos terapêuticos reuniam características clínicas e demográficas balanceadas, relacionadas a importantes fatores prognósticos da enfermidade. Assim, os pacientes das três opções terapêuticas apresentavam semelhanças quanto às condições clínicas e angiográficas, uso de medicamentos, exames laboratoriais, dentre outras - como se observa na Tabela 1, a seguir. Essas condições foram objeto de estudo da presente tese. 
Tabela 1 - Características demográficas, laboratoriais e clínicas dos pacientes

\begin{tabular}{|c|c|c|c|c|}
\hline CARACTERÍSTICA & $\begin{array}{l}\text { GRUPO } \\
\text { CLÍNICO } \\
(n=203)\end{array}$ & $\begin{array}{c}\text { GRUPO } \\
\text { ANGIOPLASTIA } \\
(n=205)\end{array}$ & $\begin{array}{c}\text { GRUPO } \\
\text { CIRURGIA } \\
(n=203)\end{array}$ & $\begin{array}{c}P \\
\left(X^{2}\right)\end{array}$ \\
\hline Idade (anos) & $60 \pm 9$ & $60 \pm 9$ & $60 \pm 9$ & 0.959 \\
\hline Sexo feminino (\%) & 31 & 33 & 28 & 0.412 \\
\hline HAS & 55 & 61 & 63 & 0.215 \\
\hline DM & 36 & 23 & 29 & 0.062 \\
\hline Tabagismo & 33 & 27 & 32 & 0.013 \\
\hline IAM prévio & 39 & 52 & 41 & 0.024 \\
\hline Angina II/III (CCS) & 78 & 78 & 86 & 0.006 \\
\hline Colesterol Total (mmol/L) & $5.74 \pm 1.01$ & $5.69 \pm 1.06$ & $5.53 \pm 1.09$ & 0.063 \\
\hline LDL- Colesterol (mmol/L) & $3.83 \pm 0.88$ & $3.80 \pm 0.93$ & $3.70 \pm 0.93$ & 0.305 \\
\hline HDL- Colesterol (mmol/L) & $0.96 \pm 0.26$ & $0.98 \pm 0.26$ & $0.96 \pm 0.26$ & 0.870 \\
\hline Triglicerídeo (mmol/L) & $2.01 \pm 0.93$ & $2.04 \pm 0.82$ & $1.91 \pm 0.95$ & 0.235 \\
\hline $\begin{array}{l}\text { Teste Ergométrico } \\
\text { positivo (\%) }\end{array}$ & 47 & 33 & 56 & 0.705 \\
\hline FEVE (\%) & $68 \pm 7$ & $67 \pm 8$ & $67 \pm 9$ & 0.984 \\
\hline Biarterial (\%) & 41 & 42 & 42 & 0.980 \\
\hline Triarterial (\%) & 59 & 58 & 58 & 0.980 \\
\hline $\begin{array}{l}\text { DAC obstrutiva em ACDA } \\
\text { proximal }\end{array}$ & 89 & 93 & 93 & 0.312 \\
\hline
\end{tabular}

$P$ - Nível estatístico de significância; HAS - Hipertensão Arterial Sistêmica; DM - Diabetes Mellitus; IAM - Infarto Agudo do Miocárdio; Angina II/III (CCS) - Angina Pectoris classe II ou III pela Canadian Cardiovascular Society; FEVE - Fração de Ejeção Ventricular Esquerda; DAC obstrutiva em ACDA - Doença Arterial Coronariana obstrutiva em Artéria Coronária Descendente Anterior. 
Todos os pacientes tiveram abordagens clínicas conforme definição prévia. Não houve falha no seguimento de nenhum deles. A duração mínima de seguimento foi de cinco anos. Os eventos cardíacos adversos ao longo desse período já foram publicados anteriormente, e podem ser observados na Tabela 2.

Tabela 2 - Eventos cardiovasculares adversos - 5 anos de seguimento

\begin{tabular}{lcccc}
\hline $\begin{array}{l}\text { DESFECHO } \\
\text { CLÍNICO }\end{array}$ & $\begin{array}{c}\text { GRUPO } \\
\text { CLÍNICO } \\
(\mathbf{n = 2 0 3 )}\end{array}$ & $\begin{array}{c}\text { GRUPO } \\
\text { ANGIOPLASTIA } \\
(\mathbf{n = 2 0 5 )}\end{array}$ & $\begin{array}{c}\text { GRUPO } \\
\text { CIRURGIA } \\
(\mathbf{n}=203)\end{array}$ & $\begin{array}{c}\boldsymbol{P} \\
\left(\boldsymbol{X}^{2}\right)\end{array}$ \\
\hline IAM (\%) & $44(21.7)$ & $44(21.5)$ & $27(13.3)$ & 0.082 \\
\hline $\begin{array}{l}\text { Revasc. } \\
\text { Adicional (\%) }\end{array}$ & $68(33.5)$ & $85(41.5)$ & $9(4.4)$ & $<0.0001$ \\
\hline ICP (\%) & $28(13.8)$ & $64(31.2)$ & $7(3.4)$ & $<0.0001$ \\
\hline CRM (\%) & $40(19.7)$ & $21(10.2)$ & $2(0.01)$ & $<0.0001$ \\
\hline AVE (\%) & $15(7.4)$ & $12(5.9)$ & $20(9.9)$ & 0.31 \\
\hline
\end{tabular}

P - Nível estatístico de significância; IAM - Infarto Agudo do Miocárdio; Revasc. Adicional revascularização adicional; ICP - Intervenção Coronária Percutânea; CRM - Cirurgia de Revascularização do Miocárdio; AVE - Acidente Vascular Encefálico.

Existe uma diferença estatisticamente significante na incidência do desfecho primário entre esses grupos ${ }^{36}$. Os pacientes alocados no grupo CRM tiveram menor incidência do desfecho primário. Não houve diferença na mortalidade e infarto do miocárdio não fatal entre os grupos. A diferença entre os grupos foi a frequência de revascularização adicional: $3.5 \%$ dos pacientes de CRM, 24\% dos pacientes TC e 32\% dos pacientes ICP. 


\subsection{TRATAMENTO CIRÚRGICO}

Dos 203 pacientes randomizados, 198 foram submetidos a CRM, com uma média de 10 dias de internação. Foram registrados como complicações hospitalares do procedimento de revascularização, 7 episódios de acidente vascular cerebral, 4 de choque cardiogênico com uso de balão intra-aórtico, 4 de insuficiência renal aguda e hemodiálise, 1 cirurgia cardíaca por tamponamento cardíaco, 11 pneumonias nosocomiais, e 2 episódios de infecção da ferida.

Durante os cinco anos de acompanhamento, 2 pacientes necessitaram repetir a cirurgia de revascularização miocárdica e 7 pacientes necessitaram ICP (média, 1 stent por paciente). Foram registrados ainda 11 episódios de angina instável, 20 acidentes vasculares cerebrais e 32 mortes. Após a randomização, foram realizados 901 testes ergométricos, 310 ecocardiogramas, 40 cintilografias, 67 cinecoronariografias e 2.350 consultas ambulatoriais.

\subsection{TRATAMENTO CLÍNICO}

Foram randomizados para tratamento clínico 203 pacientes. Após cinco anos de seguimento, 40 foram submetidos a revascularização cirúrgica (média de 11.6 dias de internação). Foram registrados como complicações 
hospitalares de procedimentos de revascularização 2 episódios de acidente vascular cerebral, 2 pneumonias nosocomiais, e 1 episódio de mediastinite. Além disso, foram realizadas 28 ICP (média de 3.7 dias de internação e 1.1 stent por paciente). Houve ainda 8 episódios de angina instável, 15 acidentes vasculares cerebrais e 35 mortes. Após a randomização, foram realizados 920 testes ergométricos, 355 ecocardiogramas, 24 cintilografias, 87 cinecoronariografias e 2458 consultas ambulatoriais.

\subsection{TRATAMENTO PERCUTÂNEO}

Dos 205 pacientes randomizados, 194 foram submetidos à ICP (média de 3.46 dias de hospitalização e 1.32 stents por paciente). Foram registrados como complicações hospitalares do procedimento de revascularização 2 episódios de acidente vascular cerebral, 2 episódios de CRM de emergência e 2 ICP adicionais de emergência. Após 5 anos de seguimento, 64 ICP adicionais foram necessárias (média de 3.1 dias de internação, e 0.78 stent por paciente). Além disso, foram realizadas 21 CRM (média de 12 dias de internação). Após o período de seguimento, houve 22 episódios de angina instável, 12 acidentes vasculares cerebrais e 28 mortes. Adicionalmente, foram feitos 988 testes ergométricos, 311 ecocardiogramas, 123 cintilografias, 138 cinecoronariografias e 2.593 consultas ambulatoriais. 


\subsection{CUSTO}

O custo cumulativo mediano do tratamento cirúrgico, após cinco anos de seguimento, foi $\mathrm{R} \$ 28.929,6$ por paciente, $\mathrm{R} \$ 12.376,8$ por paciente do grupo TC e R\$25.806,6 por paciente do ICP.

A análise pareada dos custos cumulativos mostrou diferença estatisticamente significante em favor de Tratamento Clínico (TC) contra ICP $(P<0,01)$ e CRM $(P<0,01)$; não houve diferença entre CRM e ICP. O custo cumulativo de cada grupo, com o intervalo interquatil, percentil 25 e 75\%, valor mínimo e máximo é representado na Figura 1.

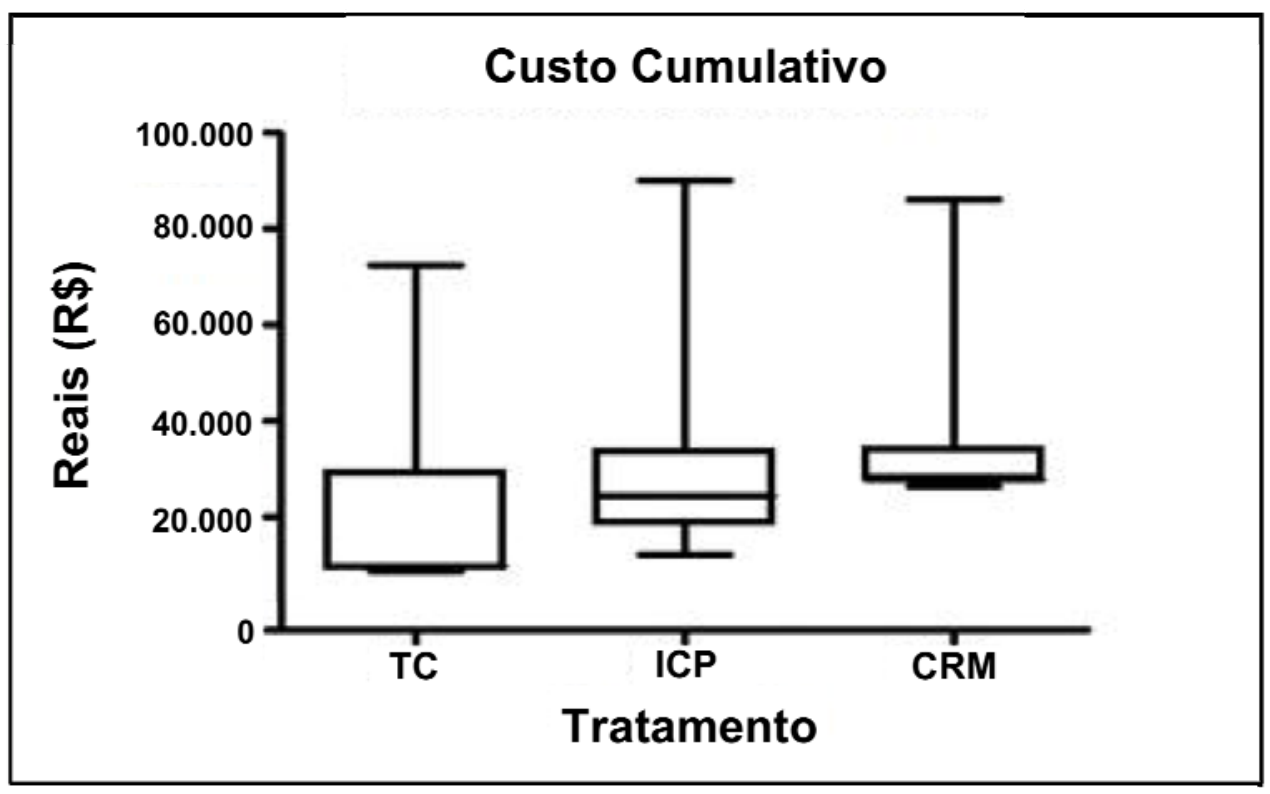

Figura 1 - Custo cumulativo das três estratégias terapêuticas

As medianas do tratamento clínico (TC) e da cirurgia de revascularização miocárdica (CRM) são iguais ao percentil 25\%. Comparação Tukey: TC $<$ ICP $<$ CRM $(P>0.0001)$; ICP e CABG $(P>0.05)$. ICP significa intervenção coronária percutânea. 
A composição dos custos das três estratégias terapêuticas, nos cinco anos de seguimento, é representada na Figura 2. Observa-se que o principal componente do custo final do Tratamento Clínico foi a terapia medicamentosa, responsável por aproximadamente $67.4 \%$ deste ao término dos cinco anos de seguimento. Em contrapartida, na ICP, o determinante foram os custos da revascularização miocárdica, seja a revascularização percutânea após a randomização ou as revascularizações adicionais, e dos exames complementares, devido a realização de coronariografias adicionais (ver Figura 2, legenda Outros). No grupo cirúrgico, o determinante do custo foi a terapia de revascularização após a randomização, responsável por $57.3 \%$ do custo cumulativo ao término dos cinco anos de seguimento.

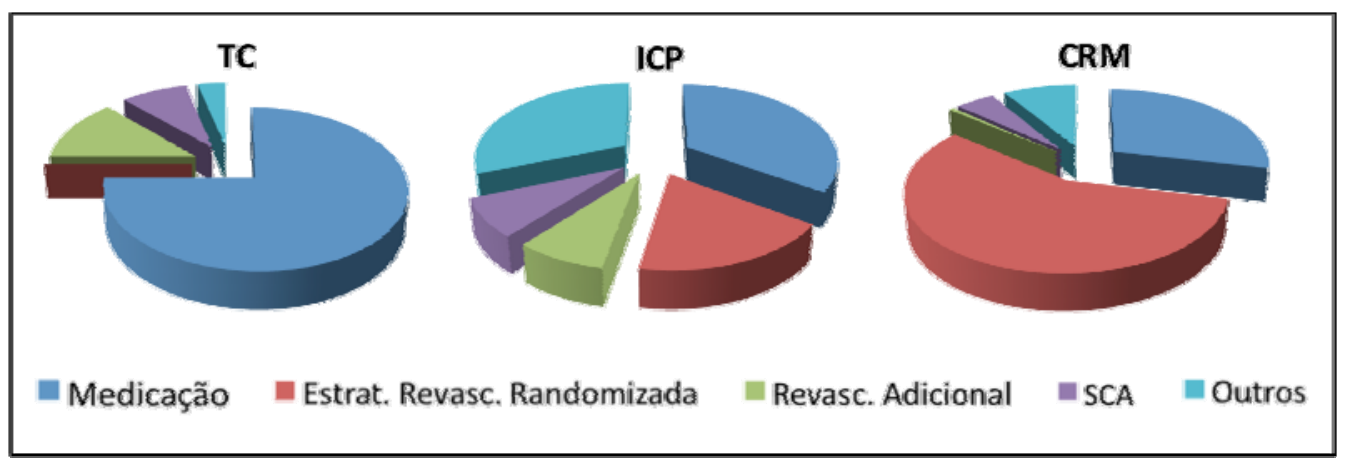

Figura 2 - Composição dos custos nas três estratégias terapêuticas

TC - Tratamento Clínico; ICP - Intervenção Coronária Percutânea; CRM Cirurgia de Revascularização Miocárdica; Estrat. Revasc. Randomizada Estratégia de Revascularização Randomizada; Revasc. Adicional Revascularização Adicional após a randomização; SCA - Síndrome Coronariana Aguda. 
A proporção do custo acumulado de cada grupo de tratamento é demonstrada na Figura 3. Observa-se ampla distribuição do custo no grupo TC. O custo de tratamento de $60 \%$ do grupo TC é inferior a $\mathrm{R} \$ 25.000,00$, enquanto o custo mínimo de tratamento do grupo CRM é superior a esse valor.

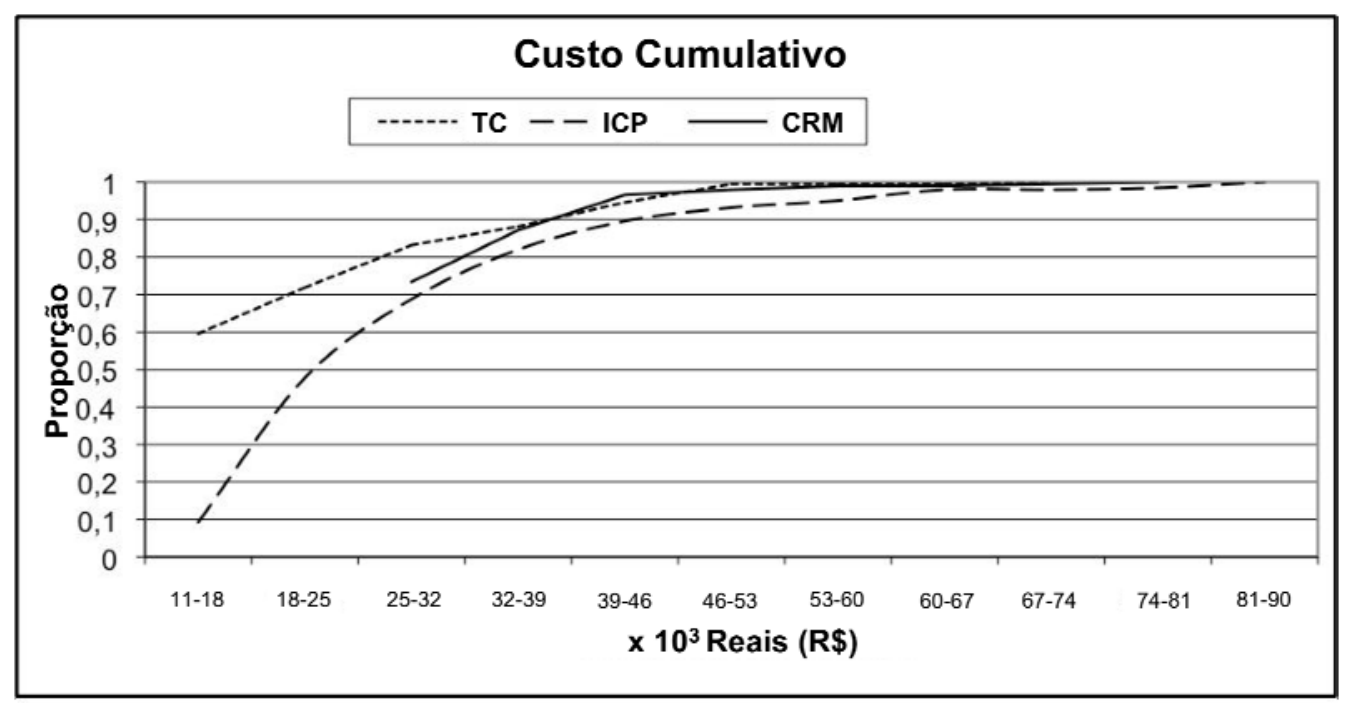

Figura 3 - Proporção do custo cumulativo das três estratégias terapêuticas

TC - Tratamento Clínico; ICP - Intervenção Coronária Percutânea; CRM Cirurgia de Revascularização Miocárdica. 


\subsection{CUSTO-EFETIVIDADE}

Para realizar a análise de custo-efetividade, como citado na metodologia, foram calculadas a sobrevida livre de evento e a proporção livre de angina, como mostra a Tabela 3.

Tabela 3 - Sobrevida livre de evento e proporção livre de angina

\begin{tabular}{lcccc}
\hline DESFECHO CLínICO & $\begin{array}{c}\text { GRUPO } \\
\text { CLÍNICO } \\
(\mathbf{n}=\mathbf{2 0 3})\end{array}$ & $\begin{array}{c}\text { GRUPO } \\
\text { ANGIOPLASTIA } \\
(\mathbf{n = 2 0 5 )}\end{array}$ & $\begin{array}{c}\text { GRUPO } \\
\text { CIRURGIA } \\
(\mathbf{n = 2 0 3 )}\end{array}$ & $\boldsymbol{P}$ \\
\hline $\begin{array}{l}\text { Tempo médio para } \\
\text { ocorrência do } \\
\text { primeiro evento, anos }\end{array}$ & 3.79 & 3.59 & 4.4 & 0.0026 * \\
Livre de angina (\%) & $111(54.8)$ & $159(77.2)$ & $151(74.2)$ & $<0.001 \dagger$ \\
\hline
\end{tabular}

*Log-rank: grupo cirúrgico apresenta um tempo mais longo, estatisticamente significante, para a ocorrência do evento.

† Teste $X^{2}$ : grupo clínico apresenta proporção menor, estatisticamente significante, de pacientes sem angina.

O tempo médio para o primeiro evento foi 3.79 anos para TC, 3.59 anos para ICP e 4.4 anos para CRM. Há uma diferença estatisticamente significante em favor dos pacientes cirúrgicos $(P=0,0026)$. Ao término do quinto ano de acompanhamento, $54.8 \%$ dos pacientes do TC não apresentavam angina. Para ICP, a proporção livre de angina foi de $77.3 \%$ e para o grupo CRM de 74.2\%, com uma diferença estatisticamente significante em comparação aos pacientes do TC $(P<0,001)$. Portanto, para sobrevida livre de eventos e sobrevida livre de eventos e angina, TC 
apresentou, respectivamente, 3.79 e 2.07 QALY; ICP apresentou 3.59 e 2.77; e CRM apresentou 4.4 e 2.81.

Ao final dos cinco anos de seguimento, os custos livres de eventos foram $\mathrm{R} \$ 16.327,80$ para $\mathrm{TC}$; $\mathrm{R} \$ 35.940,60$ para ICP e $\mathrm{R} \$ 32.873 .40$ para CRM. Os custos de cada grupo, com o intervalo interquatil, percentil 25 e 75\%, valor mínimo e máximo, são representados na Figura 4. Essas cifras indicam o custo mediano cumulativo para cada paciente, durante cinco anos de seguimento. A comparação pareada dos custos livres de eventos mostrou que houve uma diferença estatisticamente significante em favor de TC contra ICP $(P<0,01)$, e CRM $(P<0,01)$; e CRM versus ICP $(P=0,01)$.

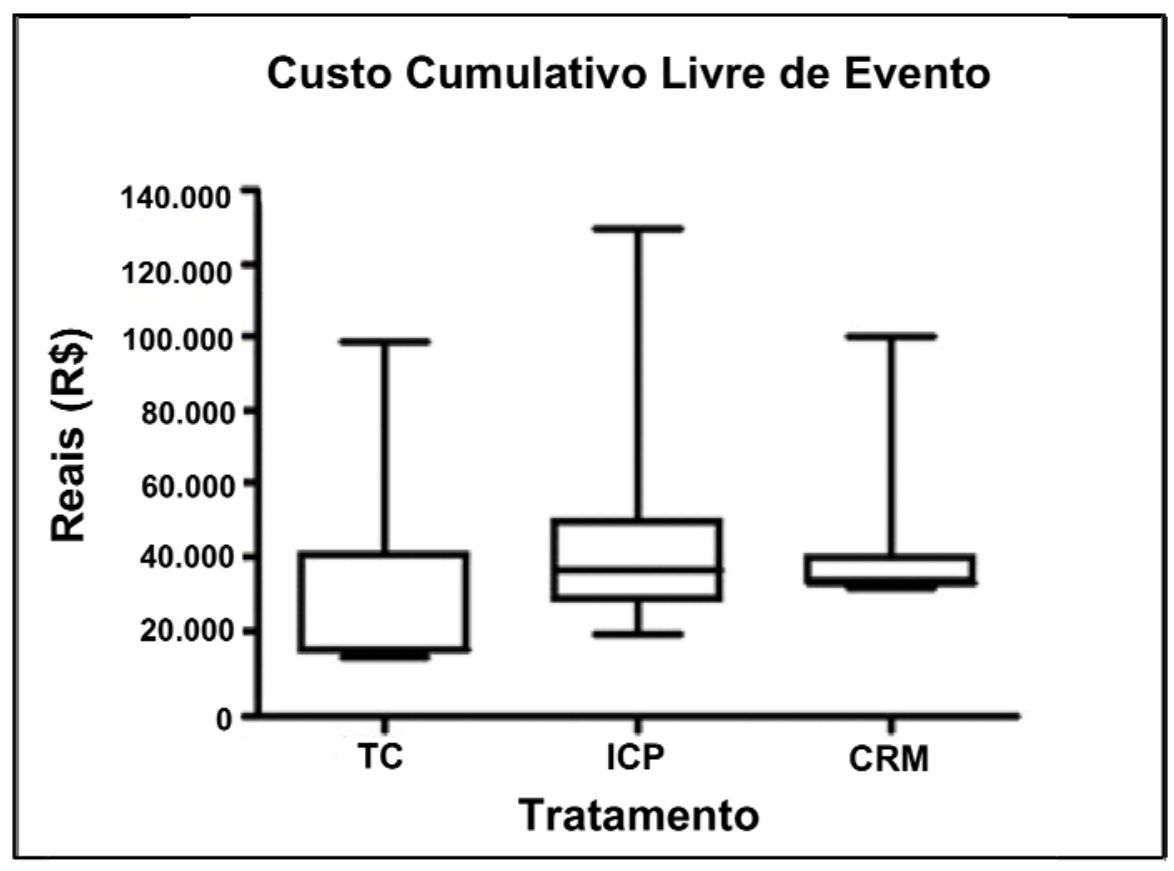

Figura 4 - Custo cumulativo livre de evento em cinco anos de seguimento As medianas do tratamento clínico (TC) e da cirurgia de revascularização miocárdica (CRM) são iguais ao percentil $25 \%$. Comparação múltipla de Tukey: TC vs ICP e CRM $(P<0.001)$; CRM vs ICP $(P=0.04)$. ICP significa intervenção coronária percutânea. 
O custo livre de eventos acrescido de livre de angina foi $R \$ 29.795,40$ para TC, $\mathrm{R} \$ 46.495,80$ para ICP e $\mathrm{R} \$ 44.305,20$ para CRM. Os custos de cada grupo, com o intervalo interquatil, percentil 25 e 75\%, valor mínimo e máximo, são representados na Figura 5. A análise pareada dos custos livres de eventos e de angina mostrou que houve uma diferença estatisticamente significante em favor de TC contra ICP $(P<0,01)$ e em comparação com CRM $(P<0,01)$; não houve diferença entre CRM e ICP.

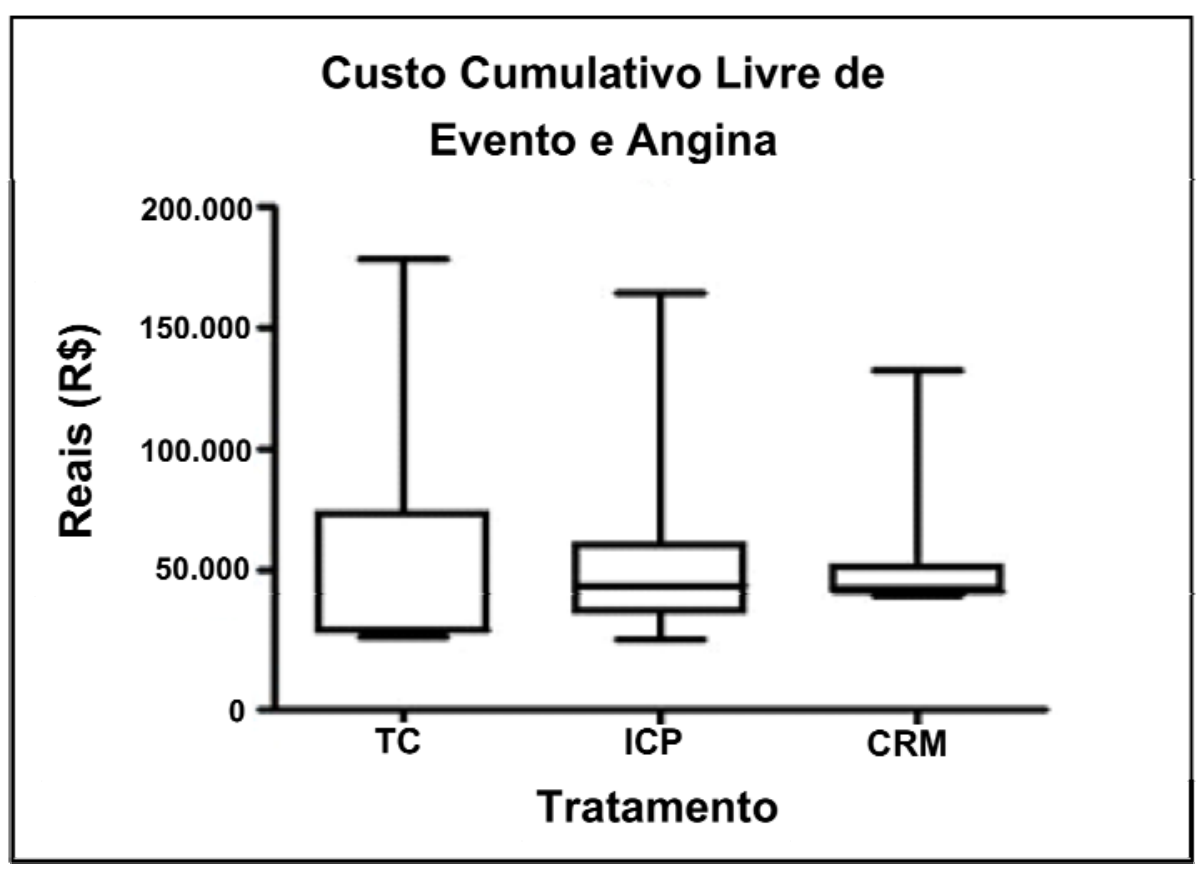

Figura 5 - Custo cumulativo livre de evento e angina em cinco anos de seguimento

As medianas do tratamento clínico (TC) e da cirurgia de revascularização miocárdica (CRM) são iguais ao percentil 25\%. Comparação múltipla de Tukey: TC vs ICP e CRM $(P<0.0001)$; ICP vs CRM $(P>0.05)$. ICP significa intervenção coronária percutânea. 


\section{Discussão}


Existem inúmeros estudos que avaliam o impacto clínico das diferentes intervenções terapêuticas descritas anteriormente na doença multiarterial coronária. Contudo, são poucos os que analisam o desfecho econômico de cada intervenção. Apenas recentemente, a análise econômica tem sido objeto de interesse dos ensaios clínicos.

As intervenções mais eficientes são aquelas que apresentam o custo mais baixo para determinado benefício em saúde. Considerando que os recursos financeiros são finitos, a análise de desfecho econômico é muito importante para as políticas de saúde, tanto públicas, quanto institucionais, contribuindo para a tomada de decisões clínicas.

O ensaio clínico MASS $\|^{36}$ revelou maior incidência de procedimentos de revascularização nos grupos TC e ICP em comparação com o grupo CRM, e pior qualidade de vida dos pacientes de TC, secundária a maior incidência de angina. Quanto à incidência de evento rígido (hard endpoint), como infarto do miocárdio ou morte, não se constatou diferença entre as três estratégias terapêuticas.

Esse é um bom cenário para comparar custo-efetividade por se tratar de um estudo randomizado que apresenta um hard endpoint semelhante. Análise de custos é uma tarefa difícil, com inúmeros vieses em potencial ${ }^{23,}$ 37-39. Para minimizar as distorções, este ensaio clínico fez uma ampla análise de custos, avaliando o custo global do tratamento de pacientes portadores 
de doença multiarterial coronária. A análise foi realizada em longo período de seguimento, contabilizando todos os recursos assistenciais: consultas ambulatoriais, medicamentos, exames complementares, hospitalização por doença cardiovascular, procedimentos de revascularização e complicação intra-hospitalar dos procedimentos de revascularização.

A análise mostrou que, na doença multiarterial coronária crônica, objetivando sobrevida livre de eventos, TC é mais econômico que CRM, e que este é mais custo-efetivo que ICP. Esse resultado é consistente com os de análises econômicas feitas por outros estudos clínicos randomizados $(\mathrm{ECR})^{40-42}$

Para a análise comparativa entre ICP e TC, destaca-se o Clinical Outcomes Utilizing Revascularization and Aggressive Drug Evaluation $(\text { COURAGE })^{43}$. Nesse estudo, comparou-se ICP $(n=1149)$ e tratamento clínico isolado otimizado $(n=1138)$, com rigoroso controle de fatores de risco cardiovascular. Após cinco anos, não se constatou diferença entre os grupos quanto à ocorrência de IAM ou morte. A necessidade de realizar a revascularização ocorreu em $21.1 \%$ na ICP (repetir a revascularização) e 32.6\% no TC $(P<0.001)$. O controle de angina foi mais eficaz nos pacientes submetidos a angioplastia em até três anos de seguimento. Aos cinco anos de seguimento não houve diferença de angina entre os grupos.

O desfecho econômico desse estudo, publicado em 2008, mostra que a adição precoce de ICP ao TC no manejo de indivíduos sintomáticos não é uma medida custo-efetiva. O custo total, ao término do estudo, foi 
estatisticamente superior (diferença líquida US\$ 10.125) no grupo ICP (US\$ 34.843 vs US\$ 24.718). O custo de medicação e de cuidados extrahospitalares foi semelhante entre os grupos. A diferença foi decorrente do custo das revascularizações, maior no grupo ICP (US\$ 14.901 vs US\$ 4.368). O tratamento clínico isolado ofereceu melhor desfecho com menor custo. O custo para melhora significativa da angina e qualidade de vida foi US\$154.580 e US\$124.233, respectivamente ${ }^{41}$.

Hambrecht e colaboradores conduziram estudo unicêntrico, randomizado, na Alemanha, comparando a intervenção coronariana percutânea ao tratamento clínico associado com atividade física diária $(20 \text { minutos/dia })^{44}$. Foram randomizados 51 pacientes para o grupo de treinamento físico e 50 para ICP. As características clínicas dos pacientes eram semelhantes (aproximadamente 95\% apresentavam angina estável CCS I ou II e 85\% eram portadores de doença coronária uni ou biarterial). Ao final do primeiro ano de seguimento, o impacto na prevenção de morte, IAM e melhora da classe funcional foi semelhante. Entretanto, os pacientes submetidos a reabilitação física apresentaram menor necessidade de revascularização ou internação hospitalar, evoluindo com maior sobrevida livre de evento (88\% versus $70 \%$ no grupo ICP, $P<0.023)$.

$\mathrm{Na}$ análise econômica desse estudo, foi contabilizada a estrutura necessária para a realização de atividade física diária (por exemplo compra de bicicleta ergométrica). O custo final do grupo ICP (US\$ 6.086) comparado ao de reabilitação física (US\$ 3.708) foi significativamente superior $(P<0.01)$. 
O gasto para promover melhora de uma classe funcional da angina (CCS1) também foi significativamente superior na ICP (US\$ 6.956) comparado à reabilitação física (US\$ 3.429). O estudo concluiu que medidas adicionadas ao tratamento clínico para proporcionar redução em revascularização são custo-efetivas $^{44}$.

Outro ensaio clínico, The Second Randomized Intervention Treatment of Angina (RITA-2), revelou um custo médio adicional de 2.685 libras esterlinas por paciente randomizado para ICP durante um período de três anos de seguimento ${ }^{42}$.

A publicação mais recente nesse assunto é proveninente do estudo BARI 2D (Bypass Angioplasty Revascularization Investigation 2 Diabetes) ${ }^{45}$. Hlatky e colaboradores publicaram, em dezembro de 2009, a análise desse grande estudo multicêntrico, composto por 2.005 pacientes portadores de diabetes tipo 2 que foram randomizados para tratamento clínico $(n=1017)$ ou terapia de revascularização miocárdica (cirúrgica ou percutânea, n=988). A técnica de revascularização foi definida pelo investigador principal de cada centro de pesquisa. A análise econômica foi realizada após quatro anos da randomização. Constatou-se que os custos médicos foram maiores no grupo submetido a revascularização comparados aos do tratamento clínico $(P<0.001)$. O custo cumulativo para o paciente cirúrgico (US\$ 80.900), em comparação com o tratamento clínico (US\$ 60.600), foi significativamente superior (US\$20.300, $P<0.0001$ ). Os pacientes submetidos a ICP também 
apresentaram custo cumulativo superior a TC (US\$ 73.400 vs US\$ 67.800, $P<0.02)$, em média US\$5.600 40 .

Na análise econômica comparativa de CRM e TC, há escassez de ensaio clínico randomizado. A análise realizada pelo MASS II, em um ano de seguimento, demonstrou um maior custo da CRM, embora o tratamento médico tenha apresentado maior aumento de custos esperados (317\% vs 21\%) devido à menor proporção de alívio de angina ${ }^{31}$. Por sua vez, a análise baseada no BARI 2D mostrou que os custos médicos foram superiores para revascularização cirúrgica do que para a terapia clínica (diferença líquida de US\$20.300, $P=0,0001)^{40}$

É interessante notar que, na análise do MASS, a vantagem econômica do tratamento clínico se manteve, mesmo considerando o fato de a população estudada apresentar doença coronária mais extensa e sintomas anginosos mais graves: aproximadamente $60 \%$ dos pacientes tinham doença coronária triarterial com envolvimento do segmento proximal da artéria descendente anterior, e aproximadamente 80\% tinham angina classe II e III, segundo a classificação da Canadian Cardiovascular Society ${ }^{29,36}$.

$\mathrm{Na}$ análise comparativa entre CRM e ICP, o BARI 2D não constatou diferença entre as duas estratégias quanto aos custos cumulativos da assistência médica após uma média de 11.4 anos de seguimento ${ }^{46}$. O custo hospitalar dos pacientes cirúrgicos foi maior. Os pacientes do grupo ICP tiveram maior necessidade de reintervenção e um custo mais elevado com terapia medicamentosa $(\mathrm{P}=0,009)$. É preciso considerar que a randomização 
desse estudo ocorreu entre agosto de 1988 e agosto de 1991, quando ainda não se dispunha do stent, e a angioplastia foi realizada apenas com balão. Devido a essa limitação metodológica, torna-se difícil uma análise econômica contemporânea ${ }^{46}$.

No estudo do ARTS, publicado em $2001^{21}$, foram randomizados 1.205 pacientes multiarteriais para serem submetidos a CRM $(n=605)$ ou ICP com stent $(n=600)$. O desfecho clínico primário foi sobrevida livre de morte, infarto agudo do miocárdio (IAM), acidente vascular encefálico (AVE) ou necessidade de repetir revascularização miocárdica ao final do primeiro ano. Após a randomização, foram realizadas 95.5\% das CRM programadas e, em média, 2.6 angioplastias por paciente (2.3 stents/paciente). Foram contabilizados os custos médicos diretos (procedimento índice, medicação, tempo de permanência hospitalar e reabilitação) para cada intervenção. Ao término do procedimento índice, o custo total da ICP em relação à CRM foi significativamente menor (US\$ 6.441 contra US\$10.653; P<0,001), uma diferença líquida total de 4.212 dólares. No entanto, devido à alta taxa de recorrência de revascularização no grupo ICP comparada à de CRM (21\% vs $3,8 \%$ ), a diferença caiu para $\cup \$ 2.973$ no primeiro ano de seguimento. Considerando-se a redução de aproximadamente 14\% na taxa de eventos combinados proporcionada pela CRM (73,8\% vs $87,8 \%)$, associada à elevada incidência de novos procedimentos de reintervenção percutânea, a limitação temporal de um ano seguimento torna-se uma restrição metodológica para a análise econômica desse estudo. 
A análise de cinco anos do MASS II reforça a idéia de que, para minimizar o viés de análise, é necessário fazer uma avaliação econômica em longo período de seguimento.

Em nossa análise de primeiro ano, o custo médio inicial de tratamento de um paciente randomizado para ICP representou aproximadamente $75 \%$ do tratamento cirúrgico. Entretanto, após 5 anos de seguimento, a revascularização cirúrgica tornou-se mais custo-efetiva para a prevenção do desfecho primário e angina.

Conclusão semelhante foi obtida no ensaio BARI: após a revascularização inicial, o custo de ICP (sem stent) foi 35\% menor do que $\mathrm{CRM}^{46}$. No entanto, ao final de cinco anos, essa diferença diminuiu para $5 \%$. Depois de 12 anos, não houve diferença entre os custos médios acumulados de ambas as estratégias de intervenção (ICP $=$ US $\$ 120,750.00$ vs CRM $=$ US\$123,000.00; $P=0,55)$.

Uma observação importante é o reduzido custo-efetividade para a melhoria da angina em pacientes com DAC extensa e sintomas graves de angina. Em comparação com TC, os pacientes dos grupos ICP e CRM experimentaram alívio significativamente maior da angina. Com isso, o custo-efetividade do TC diminuiu 82\%. Como demonstrado anteriormente, o custo estimado para a melhoria da angina é muito elevado ${ }^{41}$.

Análise econômica é uma tarefa inovadora, com dificuldades inerentes a esse processo. Um grande desafio desse tema é a determinação dos valores para a composição do custo final, ou "apropriação de custo". 
Essa dificuldade é encontrada em todos os ensaios clínicos. Observam-se valores distintos para os diversos recursos utilizados em todos os centros internacionais, tanto nas instituições americanas ${ }^{40,41,45,46}$ quanto nas européias $^{21,42,44}$.

A apropriação de custo, no presente estudo, baseou-se no sistema de saúde suplementar do Instituto do Coração do HC/FMUSP. O fato de ser prospectivo e randomizado e de a análise econômica ser comparativa entre os diversos grupos terapêuticos permite que o resultado tenha validação interna e externa, independentemente de como os custos foram apropriados $^{37,38}$. 


\section{Considerações Finais}


Embora a mensuração de custos de tratamento de doenças contenha vieses intransponíveis, a tentativa de fazer esse cálculo, ainda que de maneira aproximada, é sempre oportuna.

De fato, os custos hospitalares de determinada doença envolvem muitas variáveis, que vão desde um tratamento sem intercorrências até eventos de alta complexidade, que podem ser bastante onerosos. Nesse cenário, a cirurgia cardíaca se coloca como exemplo de procedimento com custos finais imprevisíveis.

Este estudo, cujo objetivo é mensurar os custos de três formas de tratamento de uma mesma doença, tem um caráter singular, pois, até onde sabemos, não existem na literatura análises comparativas semelhantes e de longo prazo.

Outro complicador é que nosso sistema de saúde é muito fragmentado, sendo "disputado" pelas três esferas públicas: federal, estadual e municipal. Entram ainda na disputa os gestores privados, caracterizados por Plano de saúde, Seguro-saúde, Autogestão, Cooperativas Médicas, e a Iniciativa privada.

Diante da heterogeneidade de fontes pagadoras e da diversidade de apresentações clínicas, de complicações e de insucessos terapêuticos, o cálculo dos custos revestiu-se de imensa complexidade. Outras questões a 
serem consideradas são a remuneração do pessoal, o desgaste de materiais e os insumos hospitalares.

Por fim, considerando que o estudo foi realizado em um único centro e com pessoal de assistência e pesquisa desse mesmo centro, essa análise tornou-se mais homogênea e representativa.

Este é o primeiro estudo randomizado que avalia o desfecho econômico, em longo período de seguimento, das três estratégias terapêuticas para pacientes com DAC multiarterial e função ventricular preservada. Acreditamos assim poder contribuir com informações importantes para as políticas de saúde pública ou institucionais, no sentido de otimizar os recursos disponíveis. 


\section{Conclusão}


Em conclusão, esta análise demonstrou que a adoção de estratégia de revascularização imediata na doença multiarterial coronária crônica, seja com ICP ou CRM, é menos custo-efetiva do que proporcionar um tratamento clínico otimizado, realizando o procedimento de revascularização somente quando necessário. Quando a revascularização se torna necessária, o tratamento cirúrgico é o mais custo-efetivo. 


\section{Referências}


1. American Heart Association. Heart Disease and Stroke Statistics 2009 update. Dallas Texas: American Heart Association; 2009. (2009, American Heart Association.

2. Windecker S, Maier-Rudolph W, Bonzel t, Hayricks G, Lablanche JM. Interventional cardiology in Europe 1995. Eur Heart J. 1999;20:484-95.

3. Rogers WJ for the CASS Investigators. Ten years' follow-up of quality of life in patients randomized to receive medical therapy or coronary bypass surgery study (CASS). Circulation. 1990;82:1647-58.

4. Yusuf S, Zucker D, Peduzzi P, Fischer LD, Takaro T, Kennedy JW. Effect of coronary bypass graft surgery on survival: overview of 10-year results from randomized trials by the coronary artery bypass graft surgery Trialists Collaboration. Lancet. 1994;344:563-70.

5. Davvies RF, Goldberg AD, Forman S, Pepine CJ, Knatterud GL, and Geller N. Asymptomatic Cardiac Ischemia Pilot (ACIP) study two-year followup: outcomes of patients randomized to initial strategies of medical therapy versus revascularization. Circulation. 1997;95:2037-43.

6. Pepper JR. Severe morbidity after coronary artery surgery. Eur Heart J. $1999 ; 20: 921-2$.

7. Roach G W, Kanchuger M, Mangano CM, Newman M, Nussmeier N, Wolkman R. Adverse cerebral outcomes after coronary bypass surgery. $N$ Engl J Med. 1996;335:1857-63. 
8. Gu YJ, Mariani MA, van Oeveren W, Grandgean JG, Boonstra PW. Reduction of the inflammatory response in patients undergoing minimally invasive coronary artery bypass grafting. Ann Thorac Surg. 1998;65:420-4.

9. Blauth Cl. Macroemboli and microemboli during during cardiopulmonary bypass. Ann Thorac Surg. 1995;59:1300-3.

10. Siebert J, Rogouski J, Jagielak D, Anisimowicz L, Lango R, Narkiewicz M. Atrial fibrillation after coronary artery bypass grafting without cardiopulmonary bypass. Eur J Cardiothorac Surg. 2000;17:520-3.

11. Zenati M, Domit TM, Saul M. Resource utilization for minimally invasive direct and standard coronary bypass grafting. Ann Thorac Surg. 1997;63:884-7.

12. Angelini GD, Taylor FC, Reeves BC, Ascione R. Early and mid-term outcome after off-pump and on-pump surgery in beating heart against cardioplegic arrest studies (BHACAS1 and 2): apooled analysis of two randomized controlled trials. Lancet. 2002;359:1194-9.

13. Grüntzig AR, Senning A, Siegenthaler WE. Non-operative dilatation of coronary artery stenosis: percutaneous transluminal coronary angioplasty. $N$ Engl J Med. 1979;301:61-8.

14. Hearn JA, King SB 3rd, Douglas JS Jr, Carlin SF, Lembo NJ, Ghazzal ZM. Clinical and angiographic outcomes after coronary artery stenting for acute or threatened closure after percutaneous transluminal coronary angioplasty. Initial results with a balloon-expandable, stainless steel design. Circulation. 1993;88:2086-96. 
15. Hill R, Bagust A, Bakhai A. Coronary artery stents: A rapid systematic review and economic evaluation. Heath Technol Assess. 2004;8:iii-iv,1-242.

16. Hlatky MA, Boothroyd DB, Melsop KA, Brooks MM, Mark DB, Pitt B, Reeder GS, Rogers WJ, Ryan TJ, Whitlow PL, Wiens RD. Medical costs and quality of life 10 to 12 years after randomization to angioplasty or bypass surgery for multivessel coronary artery disease. Circulation. 2004;110:19606.

17. Greenberg D, Cohen D J. Examining the economic impact of restenosis: Implications for the cost-effectiveness of an of an antiproliferative stent. Zeitschrift fur Kardiologie. 2002;3:137-43.

18. Stettler C, Wandel S, Allemann S, Kastrati A, Morice MC, Schömig A, Pfisterer ME, Stone GW, Leon MB, de Lezo JS, Goy JJ, Park SJ, Sabaté M, Suttorp MJ, Kelbaek H, Spaulding C, Menichelli M, Vermeersch P, Dirksen MT, Cervinka P, Petronio AS, Nordmann AJ, Diem P, Meier B, Zwahlen M, Reichenbach S, Trelle S, Windecker S, Jüni P. Outcomes associated with drug-eluting and bare-metal stents: a collaborative network meta-analysis. Lancet. 2007;370:937-48.

19. Bakhai A, Stone GW, Mahoney E, Lavelle TA, Shi C, Berezin RH, Lahue BJ, Clark MA, Lacey MJ, Russell ME, Ellis SG, Hermiller JB, Cox DA, Cohen DJ; TAXUS-IV Investigators. Cost effectiveness of paclitaxel-eluting stents for patients undergoing percutaneous coronary revascularization: results from the TAXUS-IV Trial. J Am Coll Cardiol. 2006;48:253-61.

20. Reynolds MR, Neil N, Ho KK, Berezin R, Cosgrove RS, Lager RA, Sirois C, Johnson RG, Cohen DJ. Clinical and economic outcomes of multivessel coronary stenting compared with bypass surgery: a single-center US experience. Am Heart J. 2003;145:334-42. 
21. Serruys PW, Unger F, Sousa JE, Jatene A, Bonnier HJ, Schönberger JP, Buller N, Bonser R, van den Brand MJ, van Herwerden LA, Morel MA, van Hout BA; Arterial Revascularization Therapies Study Group. Comparison of coronary-artery bypass surgery and stenting for the treatment of multivessel disease. N Engl J Med. 2001;344:1117-24.

22. Legrand VM, Serruys PW, Unger F, van Hout BA, Vrolix MC, Fransen GM, Nielsen TT, Paulsen PK, Gomes RS, de Queiroz e Melo JM, Neves JP, Lindeboom W, Backx B; Arterial Revascularization Therapy Study (ARTS) Investigators. Three-year outcome after coronary stenting versus bypass surgery for the treatment of multivessel disease. Circulation. 2004;109:111420.

23. Hlatky MA, Owens DK, Sanders GD. Cost-effectiveness as an outcome in randomized clinical trials. Clinical Trials. 2006;3:543-51.

24. Hlatky MA, Rogers WJ, Johnstone I, Boothroyd D, Brooks MM, Pitt B, Reeder G, Ryan T, Smith H, Whitlow P, Wiens R, Mark DB, Rosen AD, Detre $\mathrm{K}$, Frye RL; for The Bypass Angioplasty Revascularization Investigation (BARI) Investigators. Medical care costs and quality of life after randomization to coronary angioplasty or coronary bypass surgery. $N$ Engl J Med. 1997;336:92-7.

25. Elezi S, Dibra A, Folkerts U, Mehilli J, Heigl S, Schömig A, Kastrati A. Cost analysis from two randomized trials of sirolimus-eluting stents versus paclitaxel-eluting stents in high-risk patients with coronary artery disease. $J$ Am Coll Cardiol. 2006;48:262-267.

26. Girardi PB, Hueb W, Nogueira CR, Takiuti ME, Nakano T, Garzillo CL, Paulitsch Fda S, Góis AF, Lopes NH, Stolf NA. Comparative costs between 
myocardial revascularization with or without extracorporeal circulation. Arq Bras Cardiol. 2008;91:340-346.

27. Hueb W, Lopes NH, Gersh BJ, Castro CC, Paulitsch FS, Oliveira SA, Dallan LA, Hueb AC, Stolf NA, Ramires JA. A randomized comparative study of patients undergoing myocardial revascularization with or without cardiopulmonary bypass surgery: The MASS III Trial. Trials. 2008;28:52-59.

28. Favarato D, Hueb W, Gersh BJ, Soares PR, Cesar LA, da Luz PL, Oliveira SA, Ramires JA; Relative cost comparison of treatments for coronary artery disease: the First Year Follow-Up of MASS II Study. Circulation. 2003;108(Suppl 1):II21-3.

29. Hueb W, Soares PR, Gersh BJ, Cesar LA, Luz PL, Puig LB, Martinez EM, Oliveira SA, Ramires JA. The Medicine, Angioplasty, or Surgery Study (MASS II): a randomized controlled clinical trial of 3 therapeutic strategies for multivessel coronary artery disease: 1-year results. J Am Coll Cardiol. 2004;43:1743-1751.

30. Campeau L. Grading of angina pectoris (letter to the editor). Circulation. 1976;54:522-523.

31. Dodge HT, Sandler HS, Baxley WA, Hawley RR. Usefulness and limitations of radiographic methods for determining left ventricular volume. Am J Cardiol. 1966;18:10-24.

32. Serruys $P W$, Unger $F$, van Hout $B A$, van den Brand $M J$, van Herwerden LA, van Es GA, Bonnier JJRM, Simon R, Cremer J, Colombo A, Santoli C, Vandormael M, Marshall PR, Madonna O, Firth BG, Breeman A, Morel MA, Hugenholtz PG. The ARTS (Arterial Revascularization Therapies 
Study): background, goals and methods. Int J Cardiovasc Interv. 1999;2:4150.

33. National Institute for Health and Clinical Excellence. Guide to the methods of technology appraisal. www.nice.org.uk/media/B52/A7/TAMethodsGuideUpdatedJune2008.pdf

34. Nord E, Pinto JL, Richardson J, Menzel P, Ubel P. Incorporating societal concerns for fairness in numerical valuations of health programmes. Health Econ. 1999;8:25-39.

35. Coast J. Is economic evaluation in touch with society's health values? BMJ 2004; 329: 1233-1236.

36. Hueb W, Lopes NH, Gersh BJ, Soares P, Machado LA, Jatene FB, Oliveira SA, Ramires JA. Five-year follow-up of the Medicine, Angioplasty, or Surgery Study (MASS II): a randomized controlled clinical trial of 3 therapeutic strategies for multivessel coronary artery disease. Circulation. 2007;115:1082-9.

37. Cohen DJ, Reynolds MR. Interpreting the results of cost-effectiveness studies. J Am Coll Cardiol. 2008; 52:2119-26.

38. Murraya CJL, Evansa DB, Acharyab A, Baltussenc RMPM. Development of who guidelines on generalized cost-effectiveness analysis. Health Econ. 2000;9:235-51.

39. Bell CM, Urbach DR, Ray JG, Bayoumi A, Rosen AB, Greenberg D, Neumann PJ. Bias in published cost effectiveness studies: systematic review. BMJ. 2006;332:699-703. 
40. Hlatky MA, Boothroyd DB, Melsop KA, Kennedy L, Rihal C, Rogers WJ,Venkitachalam L, Brooks MM; for the Bypass Angioplasty Revascularization Investigation 2 Diabetes (BARI 2D) Study Group. Economic outcomes of treatment strategies for type 2 diabetes mellitus and coronary arterydisease in the Bypass Angioplasty Revascularization Investigation 2 Diabetes Trial. Circulation. 2009;120:2550-8.

41. Weintraub WS, Boden WE, Zhang Z, Kolm P, Zhang Z, Spertus JA, Hartigan P, Veledar E, Jurkovitz C, Bowen J, Maron DJ, O'Rourke R, Dada M, Teo KK, Goeree R, Barnett PG. Cost-effectiveness of percutaneous coronary intervention in optimally treated stable coronary patients. Circ Cardiovasc Qual Outcomes. 2008;1:12-20.

42. Sculpher MJ, Smith DH, Clayton T, Henderson RA, Buxton MJ, Pocock SJ, Chamberlain DA; Randomized Intervention Treatment of Angina (RITA-2) Trial. Coronary angioplasty versus medical therapy for angina: health service costs based on the second Randomized Intervention Treatment of Angina (RITA-2) trial. Eur Heart J. 2002;23:1291-300.

43. Boden WE, O'Rourke RA, Teo KK, Hartigan PM, Maron DJ, Kostuk WJ, Knudtson M, Dada M, Casperson P, Harris CL, Chaitman BR, Shaw L, Gosselin G, Nawaz S, Title LM, Gau G, Blaustein AS, Booth DC, Bates ER, Spertus JA, Berman DS, Mancini GB, Weintraub WS; COURAGE Trial Research Group. Optimal medical therapy with or without PCl for stable coronary disease. N Engl J Med. 2007;356:1503-16.

44. Hambrecht R, Walther C, Möbius-Winkler S, Gielen S, Linke A, Conradi K, Erbs S, Kluge R, Kendziorra K, Sabri O, Sick P, Schuler G. Percutaneous coronary angioplasty compared with exercise training in patients with stable coronary artery disease a randomized trial. Circulation. 2004;109:1371-8. 
45. Frye RL, August P, Brooks MM, Hardison RM, Kelsey SF, MacGregor JM, Orchard TJ, Chaitman BR, Genuth SM, Goldberg SH, Hlatky MA, Jones TL, Molitch ME, Nesto RW, Sako EY, Sobel BE; BARI 2D Study Group. A randomized trial of therapies for type 2 diabetes and coronary artery disease. N Engl J Med. 2009;360:2503-2515.

46. Hlatky MA, Rogers W. Medical care costs and quality of life after randomization to coronary angioplasty or coronary bypass surgery. $N$ Engl J Med. 1997;336:92-9. 